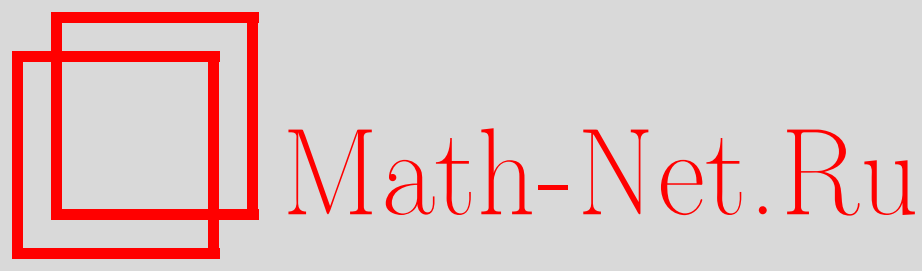

С. Ю. Доброхотов, Д. С. Миненков, С. Б. Шлосман, Асимптотика волновых функций стационарного уравнения Шредингера в камере Вейля, ТМФ, 2018, том 197, номер 2, 269-278

DOI: https://doi.org/10.4213/tmf9552

Использование Общероссийского математического портала Math-Net.Ru подразумевает, что вы прочитали и согласны с пользовательским соглашением http://www . mathnet.ru/rus/agreement

Параметры загрузки:

IP : 54.174 .149 .18

26 апреля 2023 г., 08:57:15

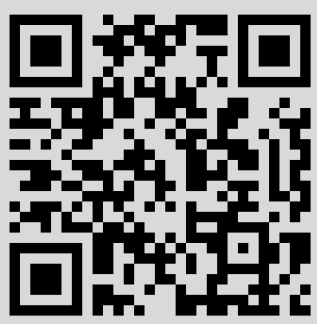




\title{
ФИЗИКА
}

Том 197, № 2

ноябрь, 2018

(C) 2018 г.

С. Ю. Доброхотов*†, Д. С. Миненков*, С. Б. Шлосман ${ }^{\ddagger}$ ף

\section{АСИМПТОТИКА ВОЛНОВЫХ ФУНКЦИЙ СТАЦИОНАРНОГО УРАВНЕНИЯ ШРЕДИНГЕРА В КАМЕРЕ ВЕЙЛЯ}

\begin{abstract}
Изучаются стационарные решения уравнения Шредингера с монотонным потенциалом $U$ в некотором многогранном угле (камере Вейля) с граничным условием Дирихле. Потенциал имеет вид $U(\mathbf{x})=\sum_{j=1}^{n} V\left(x_{j}\right), \mathbf{x}=\left(x_{1}, \ldots, x_{n}\right) \in \mathbb{R}^{n}$, с монотонно возрастающей функцией $V(y)$. Построены квазиклассические асимптотики собственных значений и собственных функций в виде определителя Слэтера, составленного из функций Эйри с нелинейно зависящими от $x_{j}$ аргументами. Предложен основанный на канонических преобразованиях способ реализации канонического оператора Маслова в виде функции Эйри.
\end{abstract}

Ключевые слова: стационарное уравнение Шредингера, краевая задача, многогранный угол типа камеры Вейля, спектр, условие квантования, канонический оператор Маслова, функции Эйри.

DOI: https://doi.org/10.4213/tmf9552

\section{1. ВВЕДЕНИЕ}

В работах [1] изучались двумерная и трехмерная модели Изинга, когда капля одной фазы соприкасается со стенкой объемлющего сосуда. Их описание потребовало рассмотрения специальной краевой задачи для $n$-мерного уравнения Шредингера

$$
-\frac{h^{2}}{2} \triangle \Psi+U(\mathbf{x}) \Psi=\mathcal{E} \Psi, \quad\left(x_{1}, \ldots, x_{n}\right) \in \mathbb{R}^{n}
$$

Работа поддержана РФФИ-СNRS (грант № 17-51-150006).

${ }^{*}$ Институт проблем механики им. А. Ю. Ишлинского РАН, Москва, Россия. E-mail: dobr@ipmnet.ru, minenkov.ds@gmail.com

${ }^{\dagger}$ Московский физико-технический институт, Долгопрудный, Московская обл., Россия

${ }^{\ddagger}$ Сколковский институт науки и технологий, Москва, Россия.

E-mail: shlosman@gmail.com

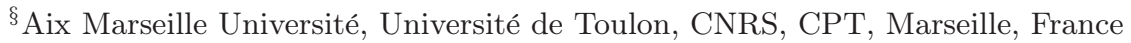

${ }^{ף}$ Институт проблем передачи информации им. А. А. Харкевича РАН, Москва, Россия 
с потенциалом $U(\mathbf{x})=\sum_{i=1}^{n} V\left(x_{i}\right), \mathbf{x}=\left(x_{1}, \ldots, x_{n}\right) \in \mathbb{R}^{n}$, с монотонно возрастающей функцией $V(x): V(0)=0, V^{\prime}(x)>0, V \in C^{2}$. Оператор Шредингера рассматривался в области $\Omega=\left\{x_{1} \geqslant x_{2} \geqslant \cdots \geqslant x_{n} \geqslant 0\right\}$ (камера Вейля) с условиями Дирихле на границе $\partial \Omega$

$$
\left.\psi\right|_{\partial \Omega}=0 .
$$

Важное наблюдение состояло в том, что собственные функции задачи (1.1), (1.2) даются определителями Слэтера. Именно, справедливо следующее утверждение.

Лемма 1. Пусть $E_{k}, \psi_{k}(x)$ - собственные значения и соответствующие собственные функции одномерной задачи

$$
-\frac{h^{2}}{2} \psi^{\prime \prime}(x)+V(x) \psi=E \psi, \quad x \geqslant 0, \quad \psi(0)=0
$$

построим определитель Слэтера, составленный из функиий $\psi_{j}\left(x_{k}\right)$ :

$$
\Psi_{\mathbf{k}}^{\mathrm{SL}}(x)=\frac{1}{n} \operatorname{det}\left(\begin{array}{ccc}
\psi_{k_{1}}\left(x_{1}\right) & \ldots & \psi_{k_{1}}\left(x_{n}\right) \\
\vdots & \ddots & \vdots \\
\psi_{k_{n}}\left(x_{1}\right) & \ldots & \psi_{k_{n}}\left(x_{n}\right)
\end{array}\right)
$$

Тогда для мультииндекса с неповторяющимися элементами $\mathbf{k}=\left\{k_{1}, \ldots, k_{n}\right\}, k_{i} \neq k_{j}$, $i, j=1, \ldots, n$, такой определитель является собственной функиией задачи (1.1), (1.2) в области $\Omega$, отвечающей собственному значению

$$
\mathcal{E}_{\mathbf{k}}=\sum_{j=1}^{n} E_{k_{j}}
$$

Этот факт справедлив только для камеры Вейля и не имеет места для общих многогранников. Данное обстоятельство указывает на наличие некоторой структуры "интегрируемости" в рассматриваемой задаче, позволяет свести изучение квазиклассической асимптотики для многомерной задачи к совокупности одномерных и, более того, для функции (потенциала) вида $V(x)=a x$ или $V(x)=a x^{2}$ построить точные решения исследуемой задачи. В первом случае собственные функции $\psi_{k}(x)$ и собственные значения $E_{k}$ одномерной задачи (1.3) выражаются через функцию Эйри и ее нули $z_{k}<0, \operatorname{Ai}\left(z_{k}\right)=0, z_{k+1}<z_{k}, k=0,1, \ldots$ :

$$
\psi_{k}=\operatorname{Ai}\left(x \sqrt[3]{\frac{2 a}{h^{2}}}+z_{k}\right), \quad E_{k}=\left|z_{k}\right| \frac{(a h)^{2 / 3}}{2^{1 / 3}} .
$$

Для других функций $V$ точные решения построить едва ли возможно и здесь можно использовать асимптотические методы. С другой стороны, как правило, квазиклассическое приближение не очень эффективно работает в краевых задачах, изменение области $\Omega$ разрушает “интегрируемость" и для краевых задач есть очень мало случаев, которые могут быть явно исследованы. Интуитивно понятно, что этот факт связан с интегрируемостью в теории биллиардов (см. [2]). Поэтому нам представляется, что в предположении, что $h$ - положительный малый параметр, рассмотрение указанной краевой задачи в камере Вейля с помощью квазиклассического приближения оказывается весьма интересным. 
В настоящей работе мы построили асимптотические решения задачи (1.1), (1.2) для произвольного монотонно растущего гладкого потенциала $V(x), V^{\prime}(x)>0$, $x>0$. В разделе 2 приведены формулы для этих решений и обсуждается связь собственных значений с условием квантования Бора-Зоммерфельда, в разделе 3 мы приводим их “геометрический” вывод, основанный на каноническом операторе Маслова и его свойствах. Для полноты изложения нужные нам свойства, касающиеся замен переменных в каноническом операторе, приведены в приложении.

\section{2. АСИМПТОТИЧЕСКИЕ СОБСТВЕННЫЕ ФУНКЦИИ И ЗНАЧЕНИЯ ОДНОМЕРНОЙ ЗАДАЧИ. УСЛОВИЯ КВАНТОВАНИЯ БОРА-ЗОММЕРФЕЛЬДА}

Рассмотрим задачу (1.3) с произвольным гладким монотонным потенциалом $V(x)$ : $V^{\prime}(x)>0, x \geqslant 0$. В этом случае асимптотики волновых функций выражаются через функции Эйри $\operatorname{Ai}(z)$, а асимптотики собственных значений выражаются через нули $\operatorname{Ai}(z)$ с помощью условий квантования, аналогичных условиям квантования Бора-Зоммерфельда. Приведем сразу соответствующие формулы, а потом их конструктивное доказательство.

Обозначим через $x^{*}(E)$ положительное решение уравнения $V(x)=E$ и введем функции

$$
\mathcal{Y}(x, E)=\operatorname{sgn}\left(x-x^{*}\right)\left|\frac{3}{2} \int_{x^{*}}^{x} \sqrt{|2(V-E)|} d x\right|^{2 / 3}
$$

И

$$
R(x, E)=\frac{\mathcal{Y}(x, E)}{V(x)-E}
$$

Нетрудно убедиться, что построенные функции $\mathcal{Y}(x, E), R(x, E)$ бесконечно дифференцируемы при $E>0$ и $\mathcal{Y}_{x}=\partial \mathcal{Y}(x, E) / \partial x$ не обращается в нуль (и, естественно, в бесконечность) на любом конечном отрезке прямой $\mathbb{R}_{x}$.

Напомним, что мы обозначили через $z_{k}, z_{k+1}<z_{k}, k=0,1, \ldots$, нули функции Эйри $\operatorname{Ai}(z)$. Определим числа $E_{k}, k=0,1,2, \ldots$, из уравнения

$$
\frac{1}{h} \int_{0}^{x^{*}\left(E_{k}\right)} \sqrt{2\left(E_{k}-V\right)} d x=\frac{2}{3}\left|z_{k}\right|^{3 / 2} .
$$

Введем функции

$$
\psi_{k}(x)=\frac{C \sqrt[4]{\left|R\left(x, E_{k}\right)\right|}}{\sqrt[6]{h}} \operatorname{Ai}\left(\frac{\mathcal{Y}\left(x, E_{k}\right)}{h^{2 / 3}}\right) .
$$

УтВЕРЖДЕНИЕ 1. 1. Функиии $\psi_{k}(x)$ и числа $E_{k}, k=0,1,2, \ldots$, определяют асимптотику собственных функций и собственных значений задачи (1.3) с точностью до $O\left(h^{5 / 6}\right)$ и $O\left(h^{2}\right)$ соответственно.

2. Виберем в определителе (1.4) функиии $\psi_{k}$ в виде (2.4) с номерами $\mathbf{k}=$ $\left(k_{1}, k_{2}, \ldots, k_{n}\right)$ и в формуле (1.5) числа $E_{k}$, найденнъе из условия квантования (2.3). Тогда эти функции и числа будут приближать собственные функции и значения оператора с точностью до $O(h)$ и $O\left(h^{2}\right)$ соответственно. 
Без условий (2.3) формула (2.4) содержится в [3], где она получена с помощью метода эталонных уравнений. Мы введем ее по-другому в разделе 3 , а сейчас обсудим равенство (2.3). Оно очевидным образом вытекает из. Напомним (см., например, [4]), что при больших отрицательных $z$ для функции Эйри справедлива асимптотическая формула

$$
\operatorname{Ai}(z)=\frac{1}{\sqrt{\pi} \sqrt[4]{-z}} \sin \left(\frac{2}{3}(-z)^{3 / 2}+\frac{\pi}{4}\right)+O\left((-z)^{3 / 2}\right) .
$$

Отсюда при условии $\mathcal{Y}\left(x, E_{k}\right) / h^{2 / 3} \ll-1$ следует, что

$$
\psi_{k}(x)=\frac{C}{\sqrt[4]{(E-V)}} \sin \left(\frac{1}{h} \int_{x^{*}}^{x} \sqrt{\left|2\left(E_{k}-V\right)\right|} d x+\frac{\pi}{4}\right)
$$

эта функция, естественно, совпадает с ВКБ-асимптотикой [5], [6] функций $\psi_{k}(x)$ слева от фокальной точки $x=x^{*}$ (вне ее окрестности). Нули этой функции определяются равенством

$$
\frac{1}{h} \int_{0}^{x^{*}\left(E_{k}\right)} \sqrt{2\left(E_{k}-V\right)} d x=\pi\left(\frac{3}{4}+k\right), \quad k=0,1,2, \ldots,
$$

которое можно переписать следующим образом. Хорошо известно (см. [6] и следующий раздел), что квазиклассические асимптотики собственных функций $\psi_{k}(x)$ связаны с кривыми на фазовой плоскости $\mathbb{R}_{p x}^{2}$ (одномерными лагранжевыми многообразиями), задаваемыми уравнениями

$$
\widetilde{\Lambda}(E)=\left\{p, x \in \mathbb{R}_{p, x}^{2}: \frac{p^{2}}{2}+V(x)=E, x \geqslant 0\right\} .
$$

В рассматриваемом случае к дуге $\widetilde{\Lambda}(E)$ разумно добавить вертикальный отрезок

$$
\Gamma(E)=\left\{p, x \in \mathbb{R}_{p, x}^{2}:-\sqrt{E-V(0)} \leqslant p \leqslant \sqrt{E-V(0)}, x=0\right\}
$$

и построить замкнутую кривую $\Lambda(E)=\widetilde{\Lambda}(E) \cup \Gamma(E)$ (см. рис. 1). Тогда равенство (2.6) можно записать в виде условия квантования Бора-Зоммерфельда

$$
\frac{1}{2 \pi} \oint_{\Lambda\left(E_{k}\right)} \sqrt{2\left(E_{k}-V\right)} d x=h\left(\frac{3}{4}+k\right) .
$$

Число $3 / 4$ есть Ind $\Lambda\left(E_{k}\right) / 4$, где Ind $\Lambda\left(E_{k}\right)$ - индекс Маслова замкнутого пути на $\Lambda\left(E_{k}\right)$, совпадающего с $\Lambda\left(E_{k}\right)$. Для гладких кривых $\Lambda\left(E_{k}\right)$ он равен 2 , но здесь кривая $\Lambda\left(E_{k}\right)$ негладкая из-за наличия граничного условия и ее индекс равен 3 . В более общем случае такого сорта многообразия ("рогатые сферы") и условия квантования на них описаны в книге [7]. Если теперь вернуться к исходной $n$-мерной задаче, ввести соответствующее $2 n$-мерное фазовое пространство, то произведение одномерных многообразий $\Lambda\left(E_{k_{j}}\right)$, отвечающих различным $x_{j}$, даст $n$-мерное негладкое лагранжево многообразие указанного типа, а условия квантования на нем дадут спектр исходного $n$-мерного оператора Шредингера.

Наконец, отметим, что условие квантования Бора-Зоммерфельда, вообще говоря, справедливо для больших $k$, а именно для $k \sim 1 / h$. Замена условия $(2.3)$ на условие квантования (2.7) фактически означает замену нулей функции Эйри на нули ее 


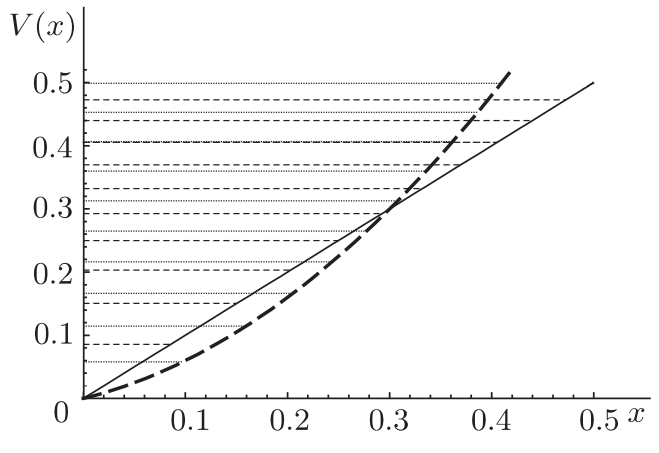

a

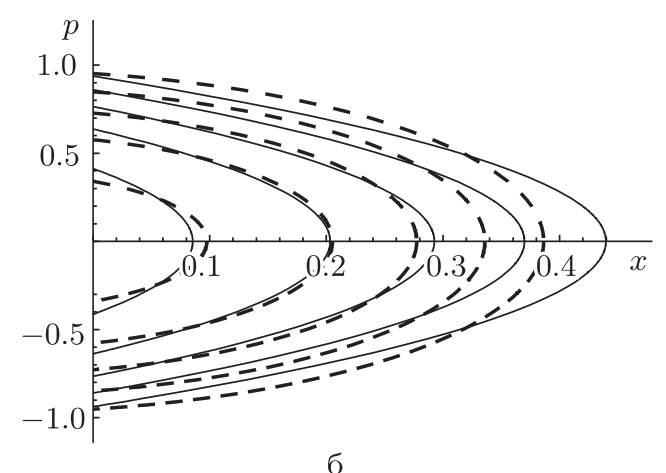

б

Рис. 1. Потенциалы $V_{1}(y)=y$ (сплошная линия) и $V_{2}=2(y+0.1)^{2}-0.02$ (штриховая линия) (а). Лагранжевы многообразия в фазовом пространстве $\Lambda_{k} \in \mathbb{R}_{p, y}^{2}$, соответствующие энергии $E_{k}, k=0,2,4,6,8$, для примера 1 (сплошные линии) и примера 2 (штриховые линии) (б).
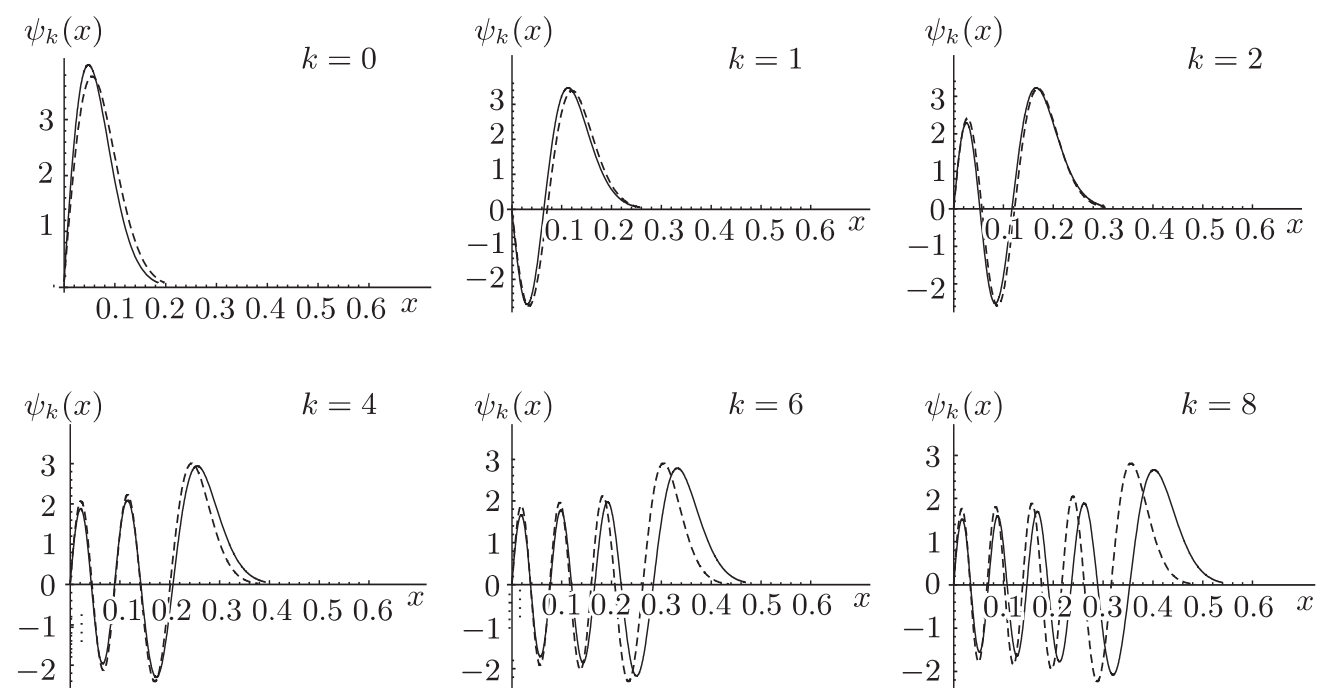

Рис. 2. Асимптотики волновых функций $\psi_{k}(x), k=0,1,2,4,6,8$, для примеров 1 (сплошные линии) и 2 (штриховые линии).

асимптотики и даже при малых $k$ дает хорошее приближение. Например, первый корень $z_{0} \approx-2.338$, а его асимптотика $\tilde{z}_{0} \approx-2.320$, т. е. ошибка меньше $1 \%$. Таким образом, при нахождении $E_{k}$ даже при $k=0,1, \ldots$ вместо условия (2.3) можно пользоваться условием (2.7).

Рассмотрим два примера:

1) $V(x)=x$

2) $V(x)=2(x+0.1)^{2}-0.02$ при $h=0.01$.

Графики потенциалов и первые 10 собственных значений представлены на рис. 1. Там же изображены соответствующие лагранжевы многообразия. 

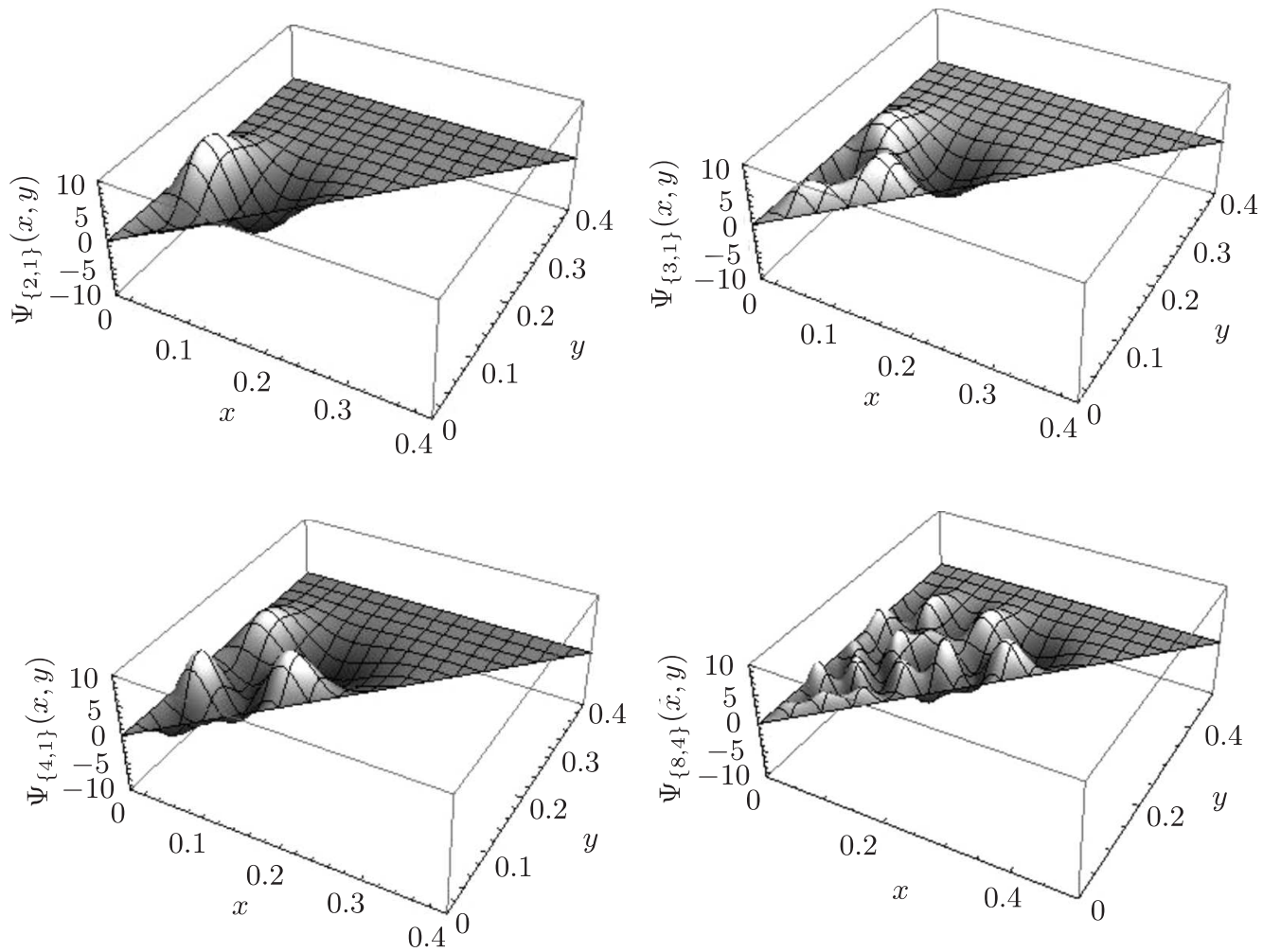

Рис. 3. Асимптотики волновых функций в угле $\Psi_{\{k, m\}}^{\mathrm{SL}}(x, y)$ для $k=2,3,4$, $m=1$ и $k=8, m=4$ для примера 2 .

На рис. 2 и рис. 3 показаны асимптотики волновых функций $\psi_{k}(x)$ и асимптотики функций $\Psi_{\{k, m\}}^{\mathrm{SL}}(x, y)$ для двумерного угла $(x, y) \in \mathbb{R}_{+}^{2}, x \leqslant y$.

\section{3. ВЫВОД АСИМПТОТИЧЕСКИХ ФОРМУЛ ДЛЯ ВОЛНОВЫХ ФУНКЦИЙ С ПОМОЩЬЮ КАНОНИЧЕСКИХ ЗАМЕН ПЕРЕМЕННЫХ В КАНОНИЧЕСКОМ ОПЕРАТОРЕ МАСЛОВА}

ДокАЗАТЕЛЬСТво утвЕРжДЕния 1. Формула (2.3) вытекает очевидным образом из (2.4). В принципе, формулы (2.4) могут быть получены с помощью метода Лангера и эталонных уравнений (см. [3], [8], а также [9]). Мы покажем здесь, как они получаются прямо из канонического оператора Маслова и его общих свойств.

Построим (формальные) асимптотики в виде канонического оператора [6], которые в одномерном случае приближают волновые функции. Асимптотики связаны с семейством (локально) инвариантных кривых - лагранжевых многообразий $\Lambda$ на фазовой плоскости $\mathbb{R}_{y, p}^{2}$, которые похожи на горизонтально расположенную параболу и являются траекториями гамильтоновой системы с гамильтонианом $H(x, p)=p^{2} / 2+V(x)$ на уровне энергии $H=E$ :

$$
\Lambda=\{p=P(t, E), x=X(t, E), t \in[-T, T]\} \equiv\{H(x, p)=E, x \geqslant 0\} .
$$


Функции $p=P(t, E), x=X(t, E)$ - решения системы Гамильтона

$$
\dot{p}=-H_{x}=-v_{x}, \quad \dot{x}=H_{p}=p,\left.\quad p\right|_{t=0}=0,\left.\quad x\right|_{t=0}=x^{*}(E) .
$$

Здесь $x^{*}(E)$ - решение уравнения $V(x)=E, x^{*}$ - точка поворота на кривых $\Lambda(E)$, время $t$ - координата на кривой - выбрано так, что значение $t=0$ соответствует точке поворота, время $t= \pm T$ соответствует точке $x=0$ оси $x$. Положительным значениям $t$ соответствуют положительные значения $P(t, E)$, а отрицательным отрицательные, при этом $P(t, E)= \pm \sqrt{E-V(X(t))}$. В качестве меры на кривых $\Lambda(E)$ естественно выбрать $d t$. Якобианы проецирования точек на кривой $\Lambda(E)$ на оси $x$ и $p$ соответственно равны $J(t, E)=\dot{X} \equiv P$ и $\widetilde{J}=\dot{P} \equiv-V_{x}$. Якобиан $J(t, E)$ обращается в нуль в точке поворота $t=0$, якобиан $\widetilde{J} \neq 0$ при всех $t$.

Многообразие $\Lambda(E)$ можно покрыть одной картой и написать асимптотику решения уравнения (1.1) (пока без учета условия Дирихле при $x=0$ ) в виде канонического оператора $K_{\Lambda(E)}^{h}$, примененного к функции, равной единице, с помощью (одного) интеграла:

$$
\begin{aligned}
\psi_{\mathrm{as}}(x, E) & =K_{\Lambda(E)}^{h} 1 \equiv \\
& \left.\equiv \frac{C}{\sqrt{2 \pi h}} \int_{-\infty}^{\infty} \frac{e(t)}{\sqrt{|\widetilde{J}(t)|}} \exp \left[\frac{i}{h}\left(\int_{0}^{t} P d X+p(x-X(t))\right)\right]\right|_{t=t(p)} d p,
\end{aligned}
$$

где $e(t)$ - гладкая срезающая функция, равная единице на отрезке $[-T, T]$ и нулю вне некоторой окрестности этого отрезка, $t(p)$ - решение уравнения $P(t)=p, C$ комплексная константа, канонический оператор $\psi_{\text {as }}$ определен с точностью $O(h)$ и не зависит от выбора разбиения единицы $e(t)$. Значение спектрального параметра $E$ выбирается из граничного условия $\psi(0, E)=0$. Недостаток этой формулы состоит в ее неэффективности, что, в частности, не позволяет получить простые формулы для спектра исходной задачи. Цель последующих рассуждений - получение эффективного представления функции (3.2) в виде функции Эйри со сложным аргументом и зависящей от $x$ амплитудой.

В принципе интеграл (3.2) можно представить в виде функции Эйри с помощью подхода, предложенного в [9], однако нам кажется, что предлагаемый ниже "геометрический" подход оказывается более простым и наглядным и, кроме того, может быть применен в других ситуациях. Именно, пусть $\Lambda$ - некоторое лагранжево многообразие на фазовой плоскости, $d \mu$ - мера, $A$ - некоторая функция на нем. Пусть в конфигурационном пространстве вместо переменной $x$ мы выбрали переменную $y=Y(x)$, причем эта замена невырожденна и для определенности $\partial Y / \partial x>0$. Эта замена индуцирует каноническую замену переменных на фазовой плоскости $x \rightarrow y$, $p \rightarrow q: y=\mathcal{Y}(x), q=p / \mathcal{Y}^{\prime}(x)$ и обратно: $x=\mathcal{X}(y), p=q / \mathcal{X}^{\prime}$. Поскольку замена координат каноническая, то для гамильтониана $H-E$ в новых координатах (обозначим его $\mathcal{H}(q, y))$ справедлива формула $\mathcal{H}(q, y, E)=H(\mathcal{P}(q, y), \mathcal{X}(y))-E$. Покажем, что замену переменных можно выбрать так, чтобы $\mathcal{H}(q, y)=g(y)\left(q^{2}+y\right)$, где $g(y)=G(\mathcal{X}(y)) \neq 0$ - гладкая функция. Действительно, имеем

$$
\mathcal{H}(q, y, E)=\left(\frac{\partial \mathcal{Y}}{\partial x}\right)^{2} \frac{q^{2}}{2}+V-E=\frac{V-E}{\mathcal{Y}}\left[\left(\frac{\partial \mathcal{Y}}{\partial x}\right)^{2} \frac{\mathcal{Y}}{2(V-E)} q^{2}+y\right]
$$


Выберем теперь функцию $\mathcal{Y}$ из условия

$$
\left(\frac{\partial \mathcal{Y}}{\partial x}\right)^{2} \frac{\mathcal{Y}}{2(V-E)}=1 \Longleftrightarrow\left(\frac{\partial \mathcal{Y}}{\partial x}\right)^{2} \mathcal{Y}=2(V-E)
$$

Добавим к этому уравнению начальное условие $\mathcal{Y}\left(x^{*}\right)=0$; интегрируя его, получим

$$
\mathcal{Y}(x, E)=\operatorname{sgn}\left(x-x^{*}\right)\left(\frac{3}{2} \int_{x^{*}}^{x} \sqrt{|2(V-E)|} d x\right)^{2 / 3} .
$$

Нетрудно убедиться, что построенная функция $\mathcal{Y}(x, E)$ бесконечно дифференцируема и $\mathcal{Y}_{x}=\partial \mathcal{Y}(x, E) / \partial x$ не обращается в нуль (и, естественно, в бесконечность) на любом конечном промежутке прямой $\mathbb{R}_{x}$. Таким образом, эта функция определяет взаимно однозначное преобразование $x \rightarrow y$, при этом

$$
R(x)=\frac{\mathcal{Y}(x)}{V(x)-E} \equiv \frac{2}{\mathcal{Y}_{x}^{2}}
$$

- также гладкая функция, не обращающаяся в нуль. Поскольку гамильтониан

$$
\mathcal{H}=\frac{\mathcal{Y}}{V-E}\left(q^{2}+y\right) \equiv R\left(q^{2}+y\right)
$$

то кривая $\Lambda$ в координатах $(q, y)$ определяется равенством

$$
\mathcal{H}=0 \quad \Longleftrightarrow \quad q^{2}+y=0
$$

Гамильтонова система в переменных $(q, y)$ с учетом равенства нулю функции $\mathcal{H}$ на траекториях принимает вид

$$
\dot{q}=-\frac{1}{R}, \quad \dot{y}=2 \frac{q}{R}
$$

Если ввести новое время $\tau$ с помощью равенства $d \tau=d t / R$, то эта система и ее решения $q=Q(\tau), y=Y(\tau)$ примут совсем простой вид (естественно, согласованный c (3.6)):

$$
\frac{d q}{d \tau}=-1, \quad \frac{d y}{d \tau}=2 q
$$

И

$$
Q=-\tau, \quad Y=-\tau^{2} .
$$

Построим теперь канонический оператор Маслова $\left[\widetilde{K}_{\Lambda(E)}^{h} A\right](y)$, действующий на функцию $A$ на кривой $\Lambda$, в новой координате $y$ в конфигурационном пространстве (координатах $(q, y)$ в фазовом пространстве) и координате $\tau$ на кривой $\Lambda$ с мерой $d \tau$. Реализуя формулу (3.2) для этого случая с учетом равенства $\tau=-q$, получим

$$
\left[\widetilde{K}_{\Lambda(E)}^{h} A\right](y)=\left.\frac{e^{i \pi / 4}}{\sqrt{2 \pi h}} \int_{-\infty}^{\infty} A(\tau) e(\tau) \exp \left[\frac{i}{h}\left(\frac{2 \tau^{3}}{3}-\tau\left(y+\tau^{2}\right)\right)\right]\right|_{\tau=-q} d q
$$

Теперь мы можем воспользоваться свойствами канонического оператора по отношению к заменам переменных. Именно, из общих свойств канонического оператора 
(см. [6], [7] и приложение) с точностью до слагаемых более высокого порядка малости по параметру $h$ (более точно до $\left.O\left(h^{5 / 6}\right)\right)$ следует равенство

$$
K_{\Lambda(E)}^{h} 1=\left.\sqrt{\left|\frac{\partial \mathcal{Y}(x)}{\partial x}\right|}\left[\tilde{K}_{\Lambda(E)}^{h} \sqrt{\left|\frac{d t}{d \tau}\right|}\right](y)\right|_{y=\mathcal{Y}(x)}=\left.\sqrt[4]{\frac{2}{R}}\left[\widetilde{K}_{\Lambda(E)}^{h} \sqrt{R}\right](y)\right|_{y=\mathcal{Y}(x)} .
$$

Заметим теперь, что функция $|R(\mathcal{X}(Y(\tau)))|$, на которую действует канонический оператор, получается из гладкой функции $|R(\mathcal{X}(y))|^{-1 / 2}$, заданной в конфигурационном пространстве. Поэтому ее можно поменять местами с каноническим оператором и получить с учетом равенств (3.3), (3.5) и определения канонического оператора

$$
\begin{aligned}
K_{\Lambda(E)}^{h} 1 & =\left.\sqrt[4]{2|R(x)|}\left[\widetilde{K}_{\Lambda(E)}^{h} 1\right](y)\right|_{y=\mathcal{Y}(x)}= \\
& =c \frac{\sqrt[4]{|R(x)|}}{\sqrt{h}} \int_{-\infty}^{\infty} e(-q) \exp \left[\frac{i}{h}\left(\frac{1}{3} q^{3}+\mathcal{Y}(x) q\right)\right] d q,
\end{aligned}
$$

$c=\sqrt[4]{2} e^{i \pi / 4} / \sqrt{2 \pi}$. Интеграл в последнем выражении с точностью до $O\left(h^{\infty}\right)$ равен $2 \pi h^{1 / 3} \operatorname{Ai}\left(\mathcal{Y}(x) / h^{2 / 3}\right)$, где $\operatorname{Ai}(y)$ - функция Эйри. Отсюда немедленно получим (2.4). Эта формула дает формальную асимптотику для волновой функции, т. е. такую, которая дает малую невязку при подстановке в исходное одномерное уравнение. Доказательство того, что (2.4) определяет "истинную” асимптотику собственной функции, практически состоит в повторении рассуждений работ [10].

\section{Замены переменных в каноническом операторе Маслова}

ПРИЛОЖЕНИЕ

Приведем здесь в компактной форме полезные для практических вычислений факты из [6], [7]. Пусть на вещественной оси $x \in \mathbb{R}$ задана замена координат $x=\mathcal{X}(y) \Longleftrightarrow y=\mathcal{Y}(x)$, причем $\partial \mathcal{X} / \partial y>0$. На фазовой плоскости $\mathbb{R}_{p, x}^{2}$ с координатами $(p, x)$ эта замена индуцирует каноническую замену переменных $(p, x) \rightarrow$ $(q, y): p=q / \mathcal{X}_{y}$. Пусть на $\mathbb{R}_{p, x}^{2}$ задана гладкая кривая (лагранжево многообразие) $\Lambda=\{p=P(\alpha), x=X(\alpha), \alpha \in \mathbb{R}\}, \alpha-$ координаты на кривой $\Lambda$. Предположим также, что на $\Lambda$ заданы другие координаты $\beta$, связанные с $\alpha$ уравнением $\alpha=\mathcal{A}(\beta) \Longleftrightarrow \beta=\mathcal{B}(\alpha), \partial \mathcal{A} / \partial \beta>0$. Наконец, предположим, что на $\Lambda$ задана функция $A(\alpha)$. Определим с помощью канонического оператора Маслова на кривой $\Lambda$ с центральной точкой $\alpha^{0}$ функцию $\psi=\left[K_{\Lambda} A(\alpha)\right](x)$. Перейдем от координат $(p, x)$ на фазовой плоскости к координатам $(q, y)$, этот переход дает на фазовой плоскости с координатами $(q, y)$ кривую $\widetilde{\Lambda}$. Выберем на $\widetilde{\Lambda}$ координату $\beta$, тогда мы можем записать $\widetilde{\Lambda}=\{q=Q(\beta), y=Y(\beta)\}$ и $\Lambda=\left\{p=P(\alpha) \equiv Q(B(\alpha)) / \mathcal{X}_{y}(Y(B(\alpha)))\right.$, $x=X(\alpha) \equiv \mathcal{X}(Y(B(\alpha)))\}$. Построим теперь канонический оператор Маслова на кривой $\widetilde{\Lambda}$ с координатами $\beta$, считая, что центральная точка $\beta^{0}=\mathcal{B}\left(\alpha^{0}\right)$. Справедливо равенство

$$
\left[K_{\Lambda} A(\alpha)\right](x)=\left.\frac{1}{\sqrt{\mathcal{X}_{y}(y)}}\left[K_{\Lambda}\left(\sqrt{\mathcal{A}_{\beta}(\beta)} A(\alpha(\mathcal{A}(\beta)))\right)\right](y)\right|_{y=\mathcal{Y}(x)} .
$$

Предположим, что в фазовом пространстве заданы гладкая функция (символ) $\Phi(p, x)$ и соответствующий ей псевдодифференциальный оператор $\Phi\left(\frac{2}{x},-i h \partial / \partial x\right)$. Подействуем этим оператором на $\left[K_{\Lambda} A(\alpha)\right](x)$, тогда справедлива формула коммутации 
псевдодифференциального оператора и канонического оператора

$$
\Phi\left(\stackrel{2}{x},-i h \frac{\frac{1}{\partial}}{\partial x}\right)\left[K_{\Lambda} A(\alpha)\right](x)=\left[K_{\Lambda}\left(\left.\Phi\right|_{\lambda} A(\alpha)+O(h)\right)\right](x),
$$

где $\left.\Phi\right|_{\lambda}$ - сужение функции $\Phi$ на $\Lambda$. Разумеется, справедлива и обратная формула, которая позволяет вынести часть амплитуды $A$ или даже всю амплитуду за канонический оператор. При этом если $\Phi$ - полином по $p$, то $\Phi(\stackrel{2}{x},-i h \partial / \partial x)-$ это дифференциальный оператор, а если $\Phi$ не зависит от $p$, то просто функция. Поэтому если в $(\Pi .1) \sqrt{\mathcal{A}_{\beta}(\beta)}$ можно представить как $\Phi(y)$, то равенство (П.1) принимает вид

$$
\left[K_{\Lambda} A(\alpha)\right](x)=\left.\frac{\Phi(y)}{\sqrt{\mathcal{X}_{y}(y)}}\left[K_{\Lambda}(A(\alpha(\mathcal{A}(\beta))))\right](y)\right|_{y=\mathcal{Y}(x)} .
$$

Это равенство означает, что мы можем построить канонический оператор Маслова, используя новые координаты $q, y$ в фазовом пространстве, вводя на лагранжевом многообразии новые координаты $\beta$, а затем перейти назад к координатам $x$ с помощью формулы $y=\mathcal{Y}(x)$. Все приведенные формулы оказываются справедливыми и в многомерном случае, если производные $\mathcal{X}_{y}(y), \mathcal{A}_{\beta}$ заменить соответственно на $\operatorname{det} \mathcal{X}_{y}(y), \operatorname{det} \mathcal{A}_{\beta}(\beta)$.

Благодарности. Авторы благодарят А.Ю. Аникина, В.Е. Назайкинского, А. И. Нейштадта и А. И. Шафаревича за обсуждения и полезные замечания.

\section{Список литературы}

[1] D. Ioffe, S. Shlosman, Y. Velenik, "An invariance principle to Ferrari-Spohn diffusions", Commun. Math. Phys., 336:2 (2015), 905-932; D. Ioffe, Y. Velenik, V. Wachtel, "Dyson Ferrari-Spohn diffusions and ordered walks under area tilts", Probab. Theory Relat. Fields, 170:1-2 (2018), 11-47.

[2] Г. Гальперин, А. Земляков, Математические биллиардь, Наука, М., 1990.

[3] С. Ю. Славянов, Асимптотика решений одномерного уравнения Шредингера, Изд-во Ленингр. ун-та, Л., 1991.

[4] М. В. Федорюк, Асимптотика. Интеграль и рядъ, Наука, М., 1987.

[5] Л. Д. Ландау, Е. М. Лифшиц, Теоретическая физика, т. 3: Квантовая механика (нерелятивистская теория), Наука, М., 2004.

[6] В.П. Маслов, Теория возмущений и асимптотические методы, Изд-во Моск. ун-та, М., 1965; В.П. Маслов, М. В. Федорюк, Квазиклассическое приближение для уравнений квантовой механики, Наука, М., 1976.

[7] М. В. Карасев, В. П. Маслов, Нелинейная скобка Пуассона. Геометрия и квантование, Наука, М., 1991.

[8] В. М. Бабич, "Математическая теория дифракции (обзор некоторых исследований, выполненных в лаборатории математических проблем геофизики ЛОМИ)", Тр. МИАН СССР, 175 (1986), 47-62; А. Найфэ, Введение в методы возмущений, Мир, М., 1984.

[9] M. V. Berry, "Uniform approximation for potential scattering involving a rainbow", Proc. Phys. Soc., 89 (1966), 479-90.

[10] Y. Colin de Verdiére, "Bohr-Sommerfeld rules to all orders", Ann. H. Poincaré, 6:5 (2005), 925-936; Д. В. Косыгин, А. А. Минасов, Я. Г. Синай, "Статистические свойства спектров операторов Лапласа-Бельтрами на поверхностях Лиувилля", УМH, 48:4(292) (1993), 3-130. 